\title{
Experiments on regulating the human body's immunity function by psychological methods
}

\author{
Yueliang Zhou ${ }^{1,}$, Chunping Zhuang ${ }^{2}$, Songqing Zhao ${ }^{3}$ \\ ${ }^{1}$ Institute of Physics Chinese Academy of Sciences, P. O. Box 603, Beijing China \\ ${ }^{2}$ Department of Industrial Engineering, Tsinghua University, Beijing 100084, China \\ ${ }^{3}$ College of Physics, Petroleum University, Beijing 102249, China
}

\section{Email address:}

moonlight-zh@163.com (Yueliang Zhou)

\section{To cite this article:}

Yueliang Zhou, Chunping Zhuang, Songqing Zhao. Experiments on Regulating the Human Body's Immunity Function by Psychological Methods. International Journal of Science, Technology and Society. Vol. 2, No. 4, 2014, pp. 91-96. doi: 10.11648/j.jists.20140204.16

\begin{abstract}
Within certain cases, the experiments on regulating the human body's immunity function by psychological methods are conducted. The results show that psychological methods can be used to drive the immunity function to eliminate or destroy abnormal cells or antigens with higher efficiency. Meanwhile, psychological methods can also be utilized to control and train the immunity function so that the healthy organs and tissues can be protected from being attacked.
\end{abstract}

Keywords: Psychological Methods, Regulating, Immunity Function

\section{Introduction}

In 1798, Edward Jenner invented the vaccination [1]. During the following several centuries, a large amount of manpower and financial resources have been put into immunology studies in the medical circle which has led to magnificent achievements [2]. Now, humans have conquered such diseases as smallpox and managed to protect or treat many infectious diseases, which has significantly stopped the spread of diseases. However, there is still a long way to go in terms of the research of immunology, both theoretically and practically.

At the beginning of the 1970s, Dr O. Carl Simonton initiated the study on the treatment of cancer with psychological methods. He carried out the linguistic guidance in which the patients were led to imagine that their immunity function is killing the cancer cells. Such an experiment gained inspirational results. It was recorded that 159 patients who were claimed to have a lifetime of no longer than one year survived over 20 months. Some patients even recovered partially or fully [3].

It was known for a long time that the psychological state significantly influences the physiological functions of human body. The effects of physiological biofeedback [4, 5], Qigong [6, 7], Yoga [8, 9], psychological hin [10, 11], hypnotherapy $[12,13]$, and meditation therapy $[14,15,16]$, are to achieve the goal of disease treatment or health care by more or less utilizing the influence of psychological methods on physiological functions of human body. Valuable achievements have been obtained by these methods.

Since the year of 1997 , we started the study on regulating the physiological functions of human body by psychological methods. The relevant reports have been published [17, 18]. To put it simply, one day in 1997, when Yueliang Zhou was taking a deep breath, a new idea came into his mind: although the lungs work automatically under normal conditions, their work is under mind control when deep breath is taken. Is it possible to control other organs, glands, tissues and functions of human body by the mind? In order to get the answer, we conducted experiments on our own bodies.

The first experiment was to regulate salivary gland secretion. The result was positive. Then more experiments were conducted, including the mind regulating the sebaceous gland, gastric peristalsis, immunity function, blood vessels state, high-density lipoprotein, recovery function of injured tissues. We found that all of those physiological functions of human body can be well regulated psychologically. Using psychological methods to regulate physiological functions of human body may be potential application value in treating some diseases. We reported the experiments in several psychology and medical conferences. Many scholars showed their interest in the 
research and carried out their own experiments. Some of them offered us the data about their experiments, which has formed the raw datums for our research. We have to admit that these experiments are fragmented, rough, and non-systematic. In addition, all of them are based on individual cases. Despite that, our research is of value as they can serve as the significant reference for large-scale and systematic experiments.

The scheme we used to treat disease by psychological methods is based on the pathological, physiological and psychological knowledge, as well as the recent physical examination reports, consists of three parts: first is to determine what the pathogeny of the diseases is, which physiological functions should be regulated to cure the disease and how to regulate those physiological functions, second is clearly explaining that mentioned above to the patients, third is conducting the practice of the scheme by the patients themselves or doctor.

The psychological methods we used to regulate the physiological functions mostly include: relaxation, focusing attention to the affected part, imagining and linguistic driving the physiological function in regulated state.

In the experiments regarding the regulation of the above mentioned functions, say the vasodilation, it takes only 3 to 10 minutes However, some other functions require a longer time to train.

There are few reports about the utilization of psychological methods to regulate the physiological functions of human body and treat diseases. Therefore, it is not surprising that some people doubt its feasibility. However, the following simple experiments, the skeptics can test it on their own.

\subsection{Treatment of Aseptic Diarrhoea, Headache Due to Intense Work, Runny Nose or Cough Due to Cold}

All these diseases can be treated with psychological methods $[17,18]$.

The method to treat the disease above, includes 3 steps:

1. relaxing,

2. concentrating attention on the affected parts,

3. imagining the smooth state of dense and hair-like blood vessels in different directions,

4. continuously murmuring: "smooth blood vessels, dense and hair-like blood vessels are smooth."

After 5 to 20 minutes, the symptoms will be relieved obviously.

\subsection{Treatment of Car Sickness}

Treating car sickness with psychological methods is as follows: concentrating attention on the stomach, imagining the state of gastric peristalsis and constantly murmuring; "relax and wriggle the stomach." 10-20 minutes later, the symptoms will be relieved to a large degree.

\subsection{Formation of Phlegm}

Normally, phlegm is beneficial to the health of human body (abnormally, phlegm is baneful). It can clean some dust, pollen, virus, bacteria and other antigens in the respiratory tract. In order to form phlegm with psychological methods, the method to produce phlegm includes 3 steps:

1. relaxing,

2. concentrating attention on respiratory tract,

3. imagining that there is phlegm on the wall of the respiratory tract,

4. continuously murmuring: "forms some phlegm."

After 5 to 15 minutes, the phlegm appears in the respiratory tract.

\section{Experiments of Utilizing Psychological Methods to Regulate the Immunity Function to Eliminate Abnormal Cells or Antigens}

Experiments on regulating the immunity function of human body to eliminate abnormal cells or antigens were conducted. Totally 35 experimenters including corn, heloma, cancer, viral cold, bacterial enteritis, and foot odor patients were clearly explained what the pathogeny of the disease is, the immunity should be regulated to cure the disease, and how to regulate immunity by psychological method.

\subsection{Treatment of Corn and Heloma}

Corn and heloma are both foot cell hyperplasia disease. They can cause pains to the patients when walking. The only difference lies in that the corn is taper shaped with even more intense pain while heloma is even hyperplasia with less intense pain. After 2 weeks of treatment with psychological methods, fourteen of the all fifteen experimenters were healed. One patient with heloma found that the heloma became smaller significantly after 8 days of treatment. Then he stopped such treatment, 12 days later, he found the heloma started growing again. Then he resumed the treatment and with ten more days passed, the heloma disappeared. Follow the steps outlined below to treat corn and heloma with psychological methods:

1. relaxing,

2. focusing attention on the affected part,

3. imagining the situation that immune cells are engulfing abnormal cells,

4. constantly murmuring: "immunity function eliminate the abnormal cells."

The treatment period lasts for 8 to 14 days, 2 times each day, 15-20 minutes for each time.

\subsection{Treatment of Cancer}

Seven patients take the experiments on using psychological methods to treat cancer, among whom 1 people recovered completely. The rest patients witnessed that the volume of cancer focus obvious reduction. The applied methods were essentially the same as which used by Dr O. Carl Simonton. The key difference lies in that the 
current treatment experiments were carried out by the patients on their own. The method is mentioned above for the treatment of corn and heloma.

One of the patients had the treatment for one of her cancer infections lasting for 40 days, each day 2 times, every time lasting for 20 minutes, and the volume of cancer focus reduced by two-fifth. While the other cancer infections were growing continuously.

After 30 days treatment using psychological method, the volume of cancer infection of one patient reduced by a third, then he gave up such treatment due to some reasons, later it was found that the volume of the cancer infection proliferated rapidly again. After 5 weeks, the focal infection exceeded the size before the treatment with psychological methods.

\subsection{Treatment of Cold}

Cold and flu may be caused by viruses [19].

Two patients catching the cold had the symptoms of fever, cough, and sore throat and so on. After 50 and 70 minutes of treatment respectively by psychological methods, the temperature returned to normal level and the coughing stopped. Afterwards, the consolidation treatment with psychological methods was carried out for 3-4 days. During the period, the treatment was carried out 3 times a day, each time lasting for $20-30$ minutes. 4 days later, the subjects recovered completely.

Follow the steps outlined below:

1. relaxing,

2. focusing on the upper respiratory tract,

3. imagining the situation that the immunity function is killing the viruses,

4. constantly murmuring: "immunity function eliminate the viruses of influenza."

Another man who had contact closely with an influenza patient, started treatment by psychological methods at the beginning of sore throat and coughing (not catching a fever). 2 times a day, 20 minutes for each time, One day later, all the symptoms disappeared. The method he used is mentioned above.

\subsection{Treatment of Enteritis}

Enteritis may be caused by germina [20,21].

A male patient, had the enteritis with diarrhea and abdominal pain, stooled for 10 times a day. After taking antibiotics, the stool frequency was reduced to about once per 8 hours, with much relieved abdominal pain, then he stopped the treatment of medicine, 5 hours later the symptoms worsened, and stool frequency increased. Under such circumstances, he started self-treatment with psychological methods according to the steps as follows:

1. relaxing,

2. focusing attention on the uncomfortable part in the abdomen,

3. imagining the situation that the immunity function is killing the bacterias,
4. constantly murmuring: “immunity function eliminate the bacteria in the intestinal tract."

Seventy minutes later, he felt that the abdominal pain was much relieved and his stool frequency was reduced to once every 6 hours. Afterwards, consolidation treatment lasting for 30 minutes was carried out once every 5 hours, in the way as mentioned above. Three days later, he recovered completely.

Two of the other enteritis patients also treated the disease by psychological method, and all of them recovered completely. The initial treating time last more than one hour, and consolidation treatment were also conducted.

\subsection{Treatment of Foot Odor}

There were 7 men who took a shower every day. However, several hours later, their feet stank. They carried out self-treatment with psychological methods according to the steps as follows:

1. relaxing,

2. focusing attention on the gaps of the toes,

3. imagining the situation that the natural killer cells are releasing toxin and killing the bacteria which releases odor,

4. constantly murmuring: "natural killer cells [22, 23] (or immunity function) eliminate the bacteria which releases odor."

They carried out 2 times of treatment every day, each time lasting for 15 to 20 minutes. 10 days later, the treatment time was reduced to 3 to 5 minutes, only one time per day. It turned out that their feet did not stink within 24 hours. Among them, one patient was conducted the following experiments: Only treated one foot with psychological method, left the other alone, The result was the foot that was left alone still stink. Then he treated both feet. As a result, both feet stop stinking.

The natural killer cells are contained in the blood, so the bacteria between the toes may be killed by the toxin released by natural killer cells. The toxin flowed along with the perspiration and reached the toes gaps.

\section{Experiments on Using Psychological Methods to Protect the Body's Normal Organs and Tissues from Being Attacked by the Immunity Function}

Under normal situation, the immunity function is supposed to protect the body's organs and tissues. However, there are many diseases which are caused by the attack of the immunity function against the body's normal organs and tissues or substances necessary to physiological function. For instance, hepatitis b [24], type I diabetes [25], glomerulonephritis [26], urticaria [27], pemphigus [28], psoriasis [29], and etc. All those diseases are difficult to cure. 
If we can use the psychological methods to realize the controlling and training of the immunity function in order to protect the body's normal organs and tissues from being attacked, then it might be a valuable option for treatment. We have carried out the experiments and gained positive results. There were totally 20 cases in the experiments, including urticaria, eczema, psoriasis, and type I diabetes samples.

First of all, all the patients should understand the pathogeny for their diseases and believe that they could utilize the psychological methods to control and train their immunity functions in order to protect the body's normal organs and tissues from being attacked.

\subsection{Treatment of Urticaria}

A male patient, more than 60 years old, and had urticarial for many years. Whenever it was cloudy or windy, he would get itchy because of the rash. He had to scratch them until the rash turned into bigger flat pustules, which became even itchier. Then the patient started the psychological treatment on the abdomen first and left the back alone. 4 weeks later, the symptoms on the abdomen were much relieved and there were no itches. However, the conditions on the back were not better. Then he repeated such treatment on his back, and another 4 weeks later, the itches on the back disappeared. The treatment steps are listed as follows:

1. relaxing,

2. focusing the attention on the itchy part of the skin,

3. constantly murmuring: "immunity function stops attacking the body's normal tissues of the skin." for 20 minutes each time, and 2 times treatment every day.

Four weeks later, the urticaria disappeared.

Six months later, the urticaria showed up again. however, the retreatment took less time, only 10 days treatment, 2 times a day, each time lasting for 15 minutes. The symptoms were gone again. The other 5 patients had similar conditions.

\subsection{Treatment of Eczema}

Five patients had eczema for years. They adopted the psychological treatment similar to that in Experiment 1. After 4-5 weeks of treatment, the skin stopped being itchy and the eczema disappeared.

\subsection{Treatment of Psoriasis}

Seven psoriasis patients adopted the psychological treatment similar to that in Experiment 1. 3-4 weeks later, the affected parts did not get itchy any longer. Another 3 weeks later, there would not be any white powder crumbs.

Five to six months later, the psoriasis symptom for four patients showed up again. However, the retreatment took less time, only about 3-7 days treatment of that mentioned above, the symptoms were gone again.

\subsection{Treatment of Diabetes}

Typical case: a male patient, about 50 years old, suffered from hyperglycemia, black skin, eyesight and physical serious decline. The researcher told him: "The cause of type I diabetes lies in the attack by the immune function against the body's pancreatic insulin-producing $\beta$ cells, and now your dark skin suggests that toxins have been transferred to the skin from within, which no longer causes the immunity function to attack the pancreas. Therefore, you will be fine." After being told such words repeatedly, the patient totally believed that the immunity function would stop attacking insulin-producing cells. Since then, as requested, he often massaged the abdomen corresponding to the pancreas while imagining and murmuring: "the immunity function no longer attack insulin-producing cells, the pancreas is healthy now and I am recovering from diabetes." 3 months later, his conditions improved markedly, with blood sugar returned to normal range and basic symptoms generally disappeared. He almost regained his health and energy.

Besides, four other patients of diabetes, have been treated by psychological methods. All the results are positive.

\section{Discussion}

Within certain cases, the experiments we conducted showed that the psychological methods can be used to drive the immunity function to eliminate the existing abnormal cells including the corn, verruca, vulgaris wart, cancer cells, bacteria, viruses, and other pathogenic antigens.

Psychological methods can be used to stimulate the immunity function and enhance the possibilities to kill the antigens to a high efficiency.

Killing the antigens at a relatively high efficient is realized by the brain work. It only works for the area where attention is paid to. It won't work for the area other than the focal point. The range of the concentrated area can be selected to a large degree, ranging from millimeters to decimeters. For instance, the treatment of urticaria can be based on a radius of 0.5 to 20 centimeters. The treatment of foot odor can be conducted on one foot or both. According to the experimental results, as the focal area enlarges, the treatment effect may be decreased. That is to say, if the focal area is too large, the effect will be influenced obviously. Normally, a radius of 2-4 centimeters is proper. If a larger area is required, focus scanning can be conducted, which means one smaller part can be chosen for treatment first and then another.

During the treatment of disease above, the abnormal cells or antigens are killed by the brain driven immunity function. Once the brain drive stops working, the killing of abnormal cells or antigens will return to a low-efficiency status. Therefore, the brain driving is indispensable for the treatment using psychological methods.

The results of the experiments we conducted show that psychological methods can be used to treat some diseases. We may guess such methods may be potential to cure some other diseases caused by antigens: more experiments are to be conducted for the examination of the guess.

The experiments within certain cases also showed that 
psychological methods can be utilized to control and train the immunity function so that the body's normal organs and tissues would not be attacked mistakenly, Therefore some disease such as psoriasis, urticaria etc could be cured.

Also the psychological methods may be potential to treat diseases such as hepatitis b, glomerulonephritis, pemphigus, and etc, because all of those diseases caused by the same pathogeny.

In the experiments, some patients of psoriasis and urticaria, suffered from the relapse, the immunity function attacked the body's normal tissues again after the disease was cured several months. We also noticed that the time for treatment again, was significantly shorter than the initial treatment time. So if the patients using a short time to conduct the treatment before the relapse, there may be no relapse completely. Experiments are necessary to examine such guess Enhance the consolidation treatment may be another speculation approach to prevent the relapse.

\section{Conclusion}

Within certain cases, we conducted some of experiments on regulating the human body's immunity function by psychological methods. As shown in the results, we can stimulate the body's immunity function with psychological methods so that they can effectively kill some antigens that we examined, including some bacteria, virus, and abnormal cells (including cancer cells). Such a high-effective state is realized and maintained by the brain-driven mechanism. Once the brain stops working, the body's immunity function will return to the state of low efficiency.

Within certain cases, we carried out the experiments on using psychological methods to control and train the human body's immunity function so that the body's normal organs and tissues would not be attacked. The results showed that we can control, train and regulate the immunity function of human body from the state of attacking the body's normal tissues to non-attacking state, the latter of which can be maintained for at least several months.

The study was carried out based on certain samples, which might be of incidence to a certain degree, so we sincerely urge all those who are interested in the study join us for further studies.

\section{Acknowledgements}

The help and support of all the participants in the study are greatly appreciated.

\section{References}

[1] Stefan Riedel, MD, PhD, "Edward Jenner and the history of smallpox and vaccination", Proc (Bayl Univ Med Cent). 18(1): pp 21-25, 2005.

[2] Arthur M. Silverstein, "History of Immunology" (Book, Second Edition, ISBN: 978-0-12-370586-0, 2009.
[3] Oscar Carl Simonton, James Creighton, Ph.D., Stéphanie Matthews Simonton, James L. Creighton, "Getting well again: a step-by-step, self-help guide to overcoming cancer for patients and their families", Bantam Book, 304 pages, April 1992.

[4] Michael, G. McKEE. "Biofeedback an overview in the context of heart-brain medicine", Cleveland Clinical Journal of Medicine, Volume 75, pp.31-34, 2008.

[5] Budzynski T.H.,Stoyva J. M. “An instrument for producing deep muscle relaxation by means of analog information feedback". Journal of applied Behavior Analysis, Volume 2, pp 231-237, 1969.

[6] Hector, W., H. Tsang. And et al., "A review on neurobiological and psychological mechanisms underlying the anti-depressive effect of Qigong exercise", Journal of Health Psychology, Volume 13, pp 857-863, 2008.

[7] Wei-Guo, Ma and Jin-Ming, Jia, "The effects and prospects of the integration of traditional Chinese medicine and Western medicine on andrology in China", Asian J Andro, Volume 13, pp 592-595, 2011.

[8] Birendranath Banerjee, H. S. Vadiraj, Amritanshu Ram. et al.,"Effects of an Integrated Yoga program in modulating psychological stress and radiation-Induced genotoxic stress in breast cancer patients undergoing radiotherapy" Integrative Cancer Therapies, Volume 6, pp 242-250, 2007.

[9] John, P. J., Sharma N, Sharma CM, KanKane A. et al.,"Effectiveness of yoga therapy in the treatment of migraine without aura: a randomized controlled trial", Headache, Volume 47, pp 654-661, 2007.

[10] S. Asch. Solomon E.,"The doctrine of suggestion, prestige and imitation in social psychology", Psychol Rev, 1 Volume. 55, pp 250-276, 1948 .

[11] Hart, J. Perry, Iondon. Oseph, T, Morris, P. Leiboyit,."EEG Alpha rhythms and susceptibility to hypnosis",Nature, Volume 219, pp 71-72, 1968.

[12] Andrew Vickers, Catherine Zollmen, "Hypnosis and therapies". West J Med. Volume 175, pp 269-272, 2001.

[13] Joseph, R. Cautel. "The use of covert conditioning in hypnotherapy", International Journal of Clinical and Experimental Hypnosi, , Volume 23, pp 15-27, 1975.

[14] Tae Sook Kim, Jeong Sook Park, Myung Ae Kim. "The relation of meditation to power and well-being", Nursing Science Quarterly, Volume 21, pp 49-58, 2008.

[15] Mark, Epstain,“Therapy and meditation”, Psychology Tody, Volume 31, pp 46-53, 1998.

[16] David, J. Kearney and Janelle, BrownChang,"Complementary and alternative medicine for IBS in adults: mind-body interventions", Nature Clinical Practice Gastroenterology \& Hepatolog, Volume 5, pp 624-636, 2008.

[17] Yueliang, Zhou. Chunping, Zhuang.Ruimin, Dong, "Application of psychological methods to control the physiological functions of the human body and treat diseases", IEEE Xplor Digital Library, 3rd International Conference on Bioinformatics and Biomedical Engineering ICBBE 2009.

[18] Yueliang, Zhou. Chunping, Zhuang.Ruimin, "Study on the Regulation of Physiological Functions of Human Body Using psychological Techniques", Review in Psychology Research, Volume 2, pp 39-46, 2013. 
[19] Ray Sahelian, Victonia Dolby Toews, "Finally...the common cold cure: Natural Remedies for Cold and Flu Mass"Paperback Book

[20] Philip, F Schofield Md, N Y Haboubi MB, D. F.Martin MB, et al., "Irritable Bowel Syndrome", Highlights in Coloproctology, .pp 112-115, 1993.

[21] Wald A. "Irritable bowel syndrome--diarrhoea. Best Pract Res Clin Gastroenterol",Volume 26(5), pp 573-80, 2012.

[22] Timmons BW, et al., "Human natural killer cell subsets and acute exercise : a brivfe review", Exerc Immunol Rev. Volume14, pp 8-23, 2008.

[23] Masilamani M, et al., "Endocytosis and intracellular trafficking of human natural killer cell receptors",. Traffic., 10(12), pp 1735-44,. 2009.

[24] P.Tiollais, P. Charnay, GN.Vysa, "Biology of Hepatitis B Virus", Science, Volume 24, pp 406-411,1981.
[25] JA Shizuru, C Taylor-Edwards, BA Banks, AK Gregory,and CG Fathman, "Immunotherapy of the nonobese diabetic mouse: treatment with an antibody to T-helper lymphocytes", Science, Volume 29, pp 659-662, 1988.

[26] A Raymond W.Steblay and Ulrich Rudofsky, "Autoimmune glomerulonephritis induced in sheep by injection of humen lung and freund's adjuvant" Science, Volume. 12, pp 204-206, 1968

[27] Clarke P, "Urticaria", Aust Fam Physicaan. Volume 33, pp 501-503, 2004

[28] AA Sinha, MT Lopez, and HO McDevitt, "Autoimmune diseases: the failure of self tolerance",. Science, Volume 15, pp 1380-1388, 1990.

[29] Gayathri K. Perera, "Integrative Biology Approach identifies Cytokine Targeting Strategies for Psoriasis" Sci Transl Med, Volume 6, pp 223, 2014. 